\title{
Reduced Complexity Optimal Hard Decision Fusion under Neyman-Pearson Criterion
}

\author{
D Nikhil
}

\author{
A Thesis Submitted to \\ Indian Institute of Technology Hyderabad \\ In Partial Fulfillment of the Requirements for \\ The Degree of Master of Technology
}

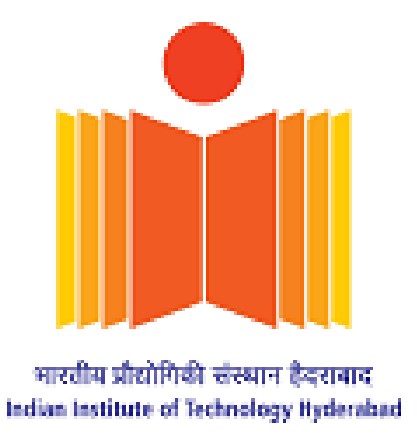

Department of Electrical Engineering 


\section{Declaration}

I declare that this written submission represents my ideas in my own words, and where ideas or words of others have been included, I have adequately cited and referenced the original sources. I also declare that I have adhered to all principles of academic honesty and integrity and have not misrepresented or fabricated or falsified any idea/data/fact/source in my submission. I understand that any violation of the above will be a cause for disciplinary action by the Institute and can also evoke penal action from the sources that have thus not been properly cited, or from whom proper permission has not been taken when needed.

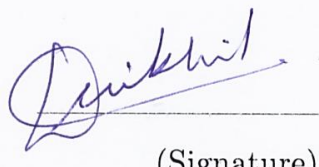

(Signature)

(D Nikhil)

EEI5MTECH 11021

(Roll No.) 


\section{Approval Sheet}

This Thesis entitled Reduced Complexity Optimal Hard Decision Fusion under Neyman-Pearson Criterion by D Nikhil is approved for the degree of Master of Technology from IIT Hyderabad

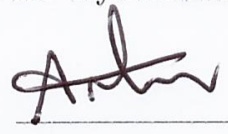

(Dr. Antony Fraklin) Examiner Dept. of CSE

IITH

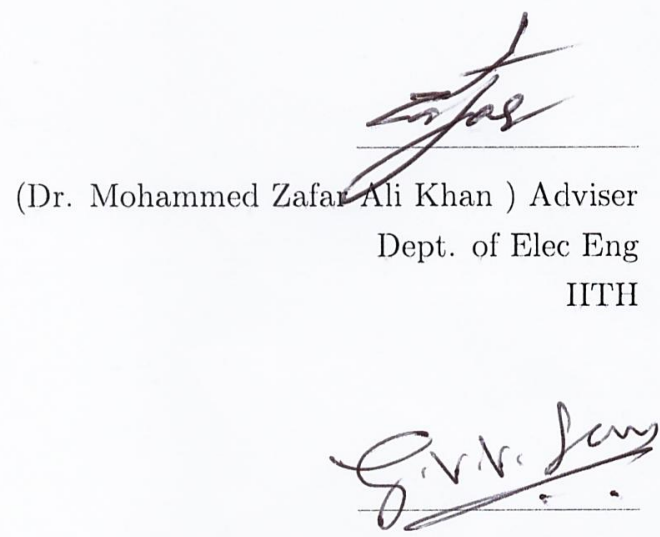

(Dr. G. V. V. Sharma) Chairman

Dept. of Elec Eng

IITH 


\section{Acknowledgements}

I would like to express my gratitude to my Supervisor Dr Mohammed Zafar Ali Khan for the useful comments, remarks, and engagement through the learning process of this master thesis. Furthermore, I would like to thank Mohammad Fayazur Rahaman for introducing me to the topic as well for the support on the way. Also, I would like to thank my friends at IIT Hyderabad, who have supported me throughout the entire process, both by keeping me harmonious and helping me putting pieces together. 


\section{Dedication}

I would like to dedicate my thesis to my parents,my teachers and my friends who inspired me to be a part of my success. 


\begin{abstract}
Distributed detection is an important part of many of the applications like wireless sensor networks, cooperative spectrum sensing in the cognitive radio network. Traditionally optimal non-randomized hard decision fusion rule under Neyman Pearson(NP) criterion is exponential in complexity. But recently [4] this was solved using dynamic programming. As mentioned in [4] that decision fusion problem exhibits semi-monotonic property in a special case. We use this property in our simulations and eventually apply dynamic programming to solve the problem with further reduced complexity. Further, we study the effect of using multiple antennas at FC with reduced complexity rule.
\end{abstract}




\section{Contents}

Declaration ...................................... ii

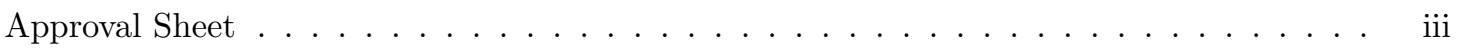

Acknowledgements . . . . . . . . . . . . . . . . . . . iv iv

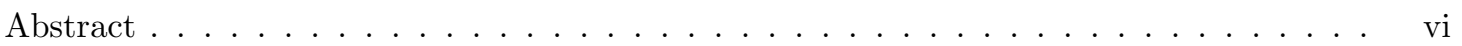

$\begin{array}{lc}\text { Nomenclature } & \text { vii }\end{array}$

1 Introduction $\quad 1$

2 Reduced Complexity Optimal Hard Decision Fusion under Neyman-Pearson Criterion 3

2.1 System model . . . . . . . . . . . . . . . . . . . . . . . . . 3

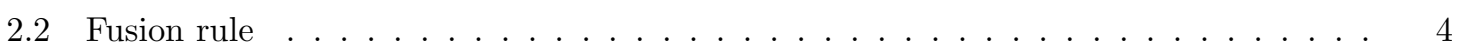

2.2.1 Dynamic programing . . . . . . . . . . . . . . . 5

2.2 .2 Branch and Bound . . . . . . . . . . . . . . . . . . 6

2.2 .3 Likelihood ratio test . . . . . . . . . . . . . . . . . . 7

2.3 Semi -Monotonic Property . . . . . . . . . . . . . . . . . . . . 8

2.4 Variable Reduction in GDFP . . . . . . . . . . . . . . . . . . . . . . . . 10

3 Numerical Results $\quad 12$

4 Performance improvement with Reduced Complexity Optimal Hard Decision Fu$\begin{array}{lr}\text { sion under NP Criterion } & 14\end{array}$

4.1 Maximal Ratio Combing . . . . . . . . . . . . . . . . . . . . . . . 15

4.2 Alamouti code . . . . . . . . . . . . . . . . . . . . 16

$4.32 \mathrm{x} 1$ Alamouti Code . . . . . . . . . . . . . . . . . . . 16

$4.42 \mathrm{2} 2$ Alamouti Code . . . . . . . . . . . . . . . . . . . . 17

5 Numerical Results $\quad 20$

6 Conclusion $\quad 22$

$\begin{array}{lr}\text { References } & 23\end{array}$ 


\section{Chapter 1}

\section{Introduction}

In recent years Distributed detection has been the widely researched topic due to the low cost of sensors and increased computational capabilities which have probed a great research enthusiasm in this area[1],[2]. Distributed detection has got vast applications in the wireless sensor network and Cooperative Spectrum Sensing (CSS) in Cognitive Radio Networks (CRN)[3],[5]. In a CSS scheme, multiple Secondary Users (SUs) connected via communication links to a fusion centre (FC) collaborate to increase the detection performance of the binary hypothesis test to identify the spectrum hole[6],[7],[8],[9],[10].

The Likelihood Ratio (LR) function of the SUs decisions plays a fundamental role in designing the optimal fusion rule at the FC[11]. The existence of monotonic sufficient statistic function for the LR is desirable under Neyman-Pearson(NP) criterion[11],[12]. However, many practical problems are non- monotonic wherein the optimal fusion rule requires computationally intensive exhaustive search methods for problems with multi-threshold decision equation.

Under Bayesian criterion, the computation of single threshold for LR test is straightforward when the apriori probabilities of the hypothesis and the Bayes costs are available. The computational time probability of error $\mathrm{P}_{\mathrm{E}}$ using the threshold is logarithmic and linear for monotonic and nonmonotonic problems respectively.

Under Neyman-Pearson criterion, low complexity methods like bisection, gradient descent etc., can be used to compute the optimal threshold for problems with monotonic property[13]. However, the non-monotonic problems require exhaustive search which leads to exponential increase in complexity. The complexity can be reduced by randomized test but this results in randomness in the decision equation. In[13] it is shown that the optimal solution for non-randomized decision fusion, in general, can be obtained in polynomial time by using the concepts of dynamic programming [14], [15]. Following [13], in this work, we focus on the (non- randomized) optimal hard decision fusion in the discrete observation space under Neyman-Pearson criterion[14]. The analysis in chapter 2 is taken from [4]. The main contributions are:

- We utilize local monotonic property exhibited in special case of non-monotonic decision fusion problem which reduces the dimensions of the optimal solution space.

- Applying Dynamic programming and Branch and Bound technique to obtain the solution with further reduced complexity. 
- We provide numerical comparison of the performance (ROC) and the complexity of (i) the proposed variable reduction technique and (ii) the solution of generalized decision fusion problem (GDFP) presented in [13].

- Further we would like to use the proposed method in performance enhancement of FC under erroneous channel between SUs and FC with MIMO technique 


\section{Chapter 2}

\section{Reduced Complexity Optimal Hard Decision Fusion under Neyman-Pearson Criterion}

\section{$2.1 \quad$ System model}

We consider FC with the parallel network of N distributed SUs. The SUs generate individual local binary decisions by sensing the spectrum for Primary User (PU) transmission as shown in Figure 2.1. Let $u_{i}$, denote the local binary decision of $i^{t h} \mathrm{SU}$, where $1 \leq i \leq N$. we define the following hypothesis

$$
\begin{aligned}
& H_{0}: \text { PU signal absent } \\
& H_{1}: \text { PU signal present }
\end{aligned}
$$

Thus $u_{i}=0$ implies $H_{0}$ and $u_{i}=1$ implies $H_{1}$. At FC, we receive local decisions as $\mathrm{N}$ dimenssional observational vector denoted by $\mathbf{u}$. Where $\mathbf{u}=\left[u_{1} \ldots u_{N}\right]^{T}$ which results in a discreate observational space $\mathcal{U}$ with cardinality $M=2^{N}$. The $m^{t h}$ vector in the observation space is represented as $\mathbf{u}_{m}, m \in\{0, \ldots, M-1\}$.

Let $p_{d_{i}}$ denote average probability of detection of $i^{t h}$ SU. Then $p_{d_{i}}=P_{r}\left\{u_{i}=1 \mid H_{1}\right\}$ similarly $p_{f_{i}}$ denotes average probability of false alarm of $i^{t h} \mathrm{SU}$. Then $p_{f_{i}}=P_{r}\left\{u_{i}=0 \mid H_{1}\right\}$.

Assuming the local decisions are independent, the conditional probabilities of $\mathbf{u}_{m}$ under each hypothesis is given by

$$
\begin{aligned}
p\left(\mathbf{u}_{m} \mid H_{1}\right) & =\prod_{i=0}^{N-1} p_{d_{i}}^{u_{i}}\left(1-p_{d_{i}}\right)^{\left(1-u_{i}\right)} \\
p\left(\mathbf{u}_{m} \mid H_{0}\right) & =\prod_{i=0}^{N-1} p_{f_{i}}^{u_{i}}\left(1-p_{f_{i}}\right)^{\left(1-u_{i}\right)}
\end{aligned}
$$

where $u_{i}$ is the $i^{t h}$ decision in observation vector $\mathbf{u}_{m}$. 


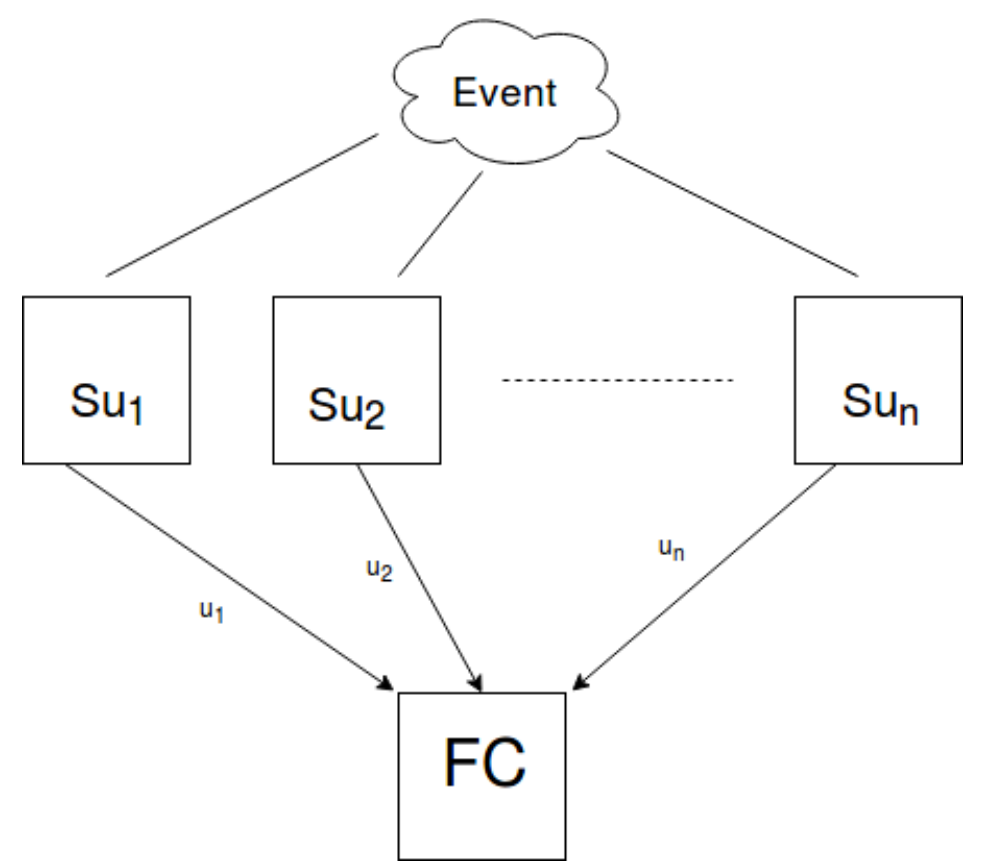

Figure 2.1: System model

\subsection{Fusion rule}

Let $\Gamma($.$) denote the fusion rule of the FC. Let u_{F C}$ denote global decision determined by FC after observing $\mathcal{U}$. Therefore $u_{F C}=\Gamma(\mathcal{U})$, where $u_{F C}=0$ implies hypothesis $H_{0}$ and $u_{F C}=1$ implies hypothesis $H_{1}$ respectively.

Let $P_{D}$ and $P_{F}$ denote probability of detection and probability of false alarm at FC that are obtained as

$$
\begin{aligned}
& P_{D}=\sum_{\mathcal{U} \in \Re 1} p\left(\mathbf{u}_{m} \mid H_{1}\right) \\
& P_{F}=\sum_{\mathcal{U} \in \Re 1} p\left(\mathbf{u}_{m} \mid H_{0}\right)
\end{aligned}
$$

where $\Re 0$ and $\Re 1$ are two decision regions in $N$-Dimensional continous real Space $R^{N}$, such that $\mathcal{U} \subset(\Re 0 \cup \Re 1), \Re 0 \cap \Re 1=\{\}$ (empty set), $\mathbf{u}_{m} \in \Re 0$ implies $\Gamma\left(\mathbf{u}_{m}\right)=0$ and $\mathbf{u}_{m} \in \Re 1$ implies $\Gamma\left(\mathbf{u}_{m}\right)=1, \forall m$. This indicates that an optimal definition of decision regions results in an optimal fusion rule.

We now formulate the Generalized Decision Fusion Problem (GDFP) as,

$$
\max _{\Re 1} P_{D}
$$

$$
\text { Sub to: } P_{F} \leq \alpha
$$

where $\alpha$ is the constrain on $P_{F}$. Under NP criterion this is a constrained optimization problem for which the solution is exponential in complexity.

Definition 1 :(0-1 Knapsack Problem (KP)). Given a set of $M$ items, each with a value and 
weight $\left\{v_{m}, w_{m}\right\}$ separately for $1 \leq m \leq M$, pick a subset $\mathrm{S}$, of items with the end goal such that

$$
\operatorname{Max} \sum_{m=1}^{M} s_{m} v_{m}, \text { sub to } \sum_{m=1}^{M} s_{m} w_{m} \leq W_{\text {lim }}
$$

where $\mathrm{S}$ is $\left[s_{1} \ldots s_{M}\right], s_{m} \in\{0,1\}, s_{m}=0$ infers the item $m$ is not picked $s_{m}=1$ infers it is picked and $W_{\text {lim }}$ is the aggregate weight confine permitted.

Here the non-randomized hard decision fusion problem is being mapped to the 0-1 Knapsack problem using (2.5), (2.6), the GDFP can be written as,

$$
\operatorname{Max} \sum_{m=1}^{M} s_{m} P_{D_{m}} \text {, sub to } \sum_{m=1}^{M} s_{m} P_{F_{m}} \leq \alpha
$$

where $P_{D_{m}}$ and $P_{F_{m}}$ are individual objective and constrained parameter of $m^{\text {th }}$ observational vector $\mathbf{u}_{m}$.

$$
\begin{aligned}
& P_{D_{m}}=p\left(\mathbf{u}_{m} \mid H_{1}\right) \\
& P_{F_{m}}=p\left(\mathbf{u}_{m} \mid H_{0}\right)
\end{aligned}
$$

By Definition $1,(2.7)$ is a $0-1 \mathrm{KP}$ where $v_{m}=P_{D_{m}}, w_{m}=P_{F_{m}}, W_{\text {lim }}=\alpha$.

\subsubsection{Dynamic programing}

It was notable that the 0-1 KP can be tackled utilizing Dynamic Programming(DP). Since DP works only on integers so we have to convert $P_{D_{m}}, P_{F_{m}}$ and $\alpha$ to integers. To perfom this operation we define scaling function as $I(a)=\lfloor r . a\rfloor$ where $a$ real-valued input argument, $r$ scaling factor which is sufficiently large. Then we define $I_{d_{m}}=I\left(P_{D_{m}}\right), I_{f_{m}}=I\left(P_{F_{m}}\right)$ and $I_{\alpha}=I(\alpha)$ Let $V(i, j)$ is the maximum value of the set of first $i$ vectors $\mathbf{u}_{m} \mathrm{~s}$ that is subject to the constraint that the sum of the $I_{f_{m}} \mathrm{~s}$ of the vectors in the set is $\leq j$. Value of the original problem corresponds to $V\left(n, I_{\alpha}\right)$ we calculate $V(i, j)$ for $1 \leq i \leq M$ and $0 \leq j \leq I_{\alpha}$ with formula (2.7).

$$
V(i, j)=\max \left(V\left(i-1, j-I_{f_{i}}\right)+I_{d_{i}}, V(i-1, j)\right)
$$

First term in (2.8) corresponds to the case when $i^{\text {th }}$ vector is included in the solution and the second term corresponds to the case when $i^{\text {th }}$ vector is not included. To know whether the vectors are included in the solution here is the step by step procedure of the algorithm.

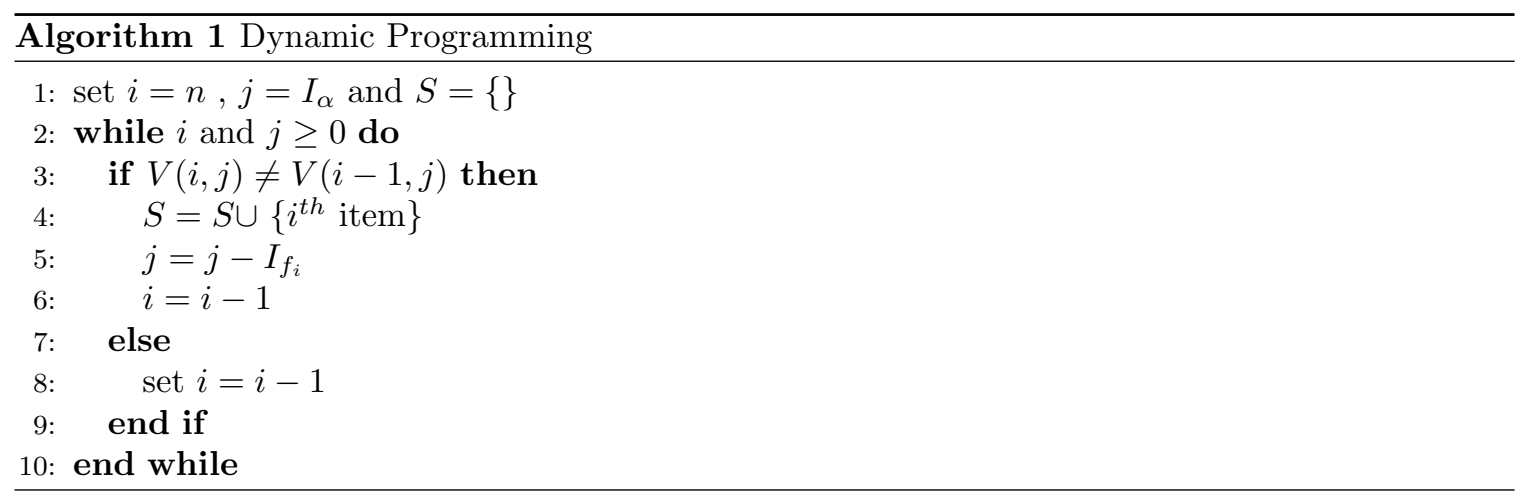


By the end of the algorithm all the items in the solution are in the set $S$.

Now let us try to understand dynamic programing with the help of example

Example: Let us try to fill the Knapsack of capacity $\mathrm{W}=5$ with items mentioned in Table 2.1

Table 2.1: List of items

\begin{tabular}{|l|l|l|}
\hline Items & Weights & Values \\
\hline 1 & 2 & 3 \\
2 & 3 & 4 \\
3 & 4 & 5 \\
4 & 5 & 6 \\
\hline
\end{tabular}

Using equation (2.8) on above data we formulate $V(i, j)$ as shown in table 2.2

Table 2.2: $V(i, j)$

Weights

\begin{tabular}{|c|c|c|c|c|c|c|c|}
\hline \multirow{6}{*}{ values } & $\downarrow i, j \rightarrow$ & 0 & 1 & 2 & 3 & 4 & 5 \\
\hline & 0 & 0 & 0 & 0 & 0 & 0 & 0 \\
\hline & 1 & 0 & 0 & 3 & 3 & 3 & 3 \\
\hline & 2 & 0 & 0 & 3 & 4 & 5 & 7 \\
\hline & 3 & 0 & 0 & 3 & 4 & 5 & 7 \\
\hline & 4 & 0 & 0 & 3 & 4 & 5 & 7 \\
\hline
\end{tabular}

Items that where put in the knapsack are found using Algorithm 1. Finally the items in Knapsack are $\mathrm{S}=\{1,2\}$.

\subsubsection{Branch and Bound}

Here is another approach for solving 0-1 KP which uses the state-space-tree which comprises an initial state, final state and intermediate states[?].

In state space tree each node consist of level, $P_{D_{m}}, P_{F_{m}}$ and bound. The step by step procedure of algorithm is given in Algorithm 2.

In above algorithm, we have considered algorithm reset_u where $\mathrm{u}$ is a node. The steps involved in the algorithm reset_u are as follows.

In reset_u Bound is a Greedy solution which is used to find the bound on the maximum $P_{D_{m}}$. All the mark item form the solution for GDFP.

Now let us try to understand Branch and Bound algorithm with the help of an example

Example: Let us try to fill the Knapsack of capacity W=16 with items mentioned in Table 2.1

Table 2.3: List of example

\begin{tabular}{|l|l|l|}
\hline Items & Weights & Values \\
\hline 1 & 3 & 45 \\
2 & 5 & 30 \\
3 & 9 & 45 \\
4 & 5 & 10 \\
\hline
\end{tabular}



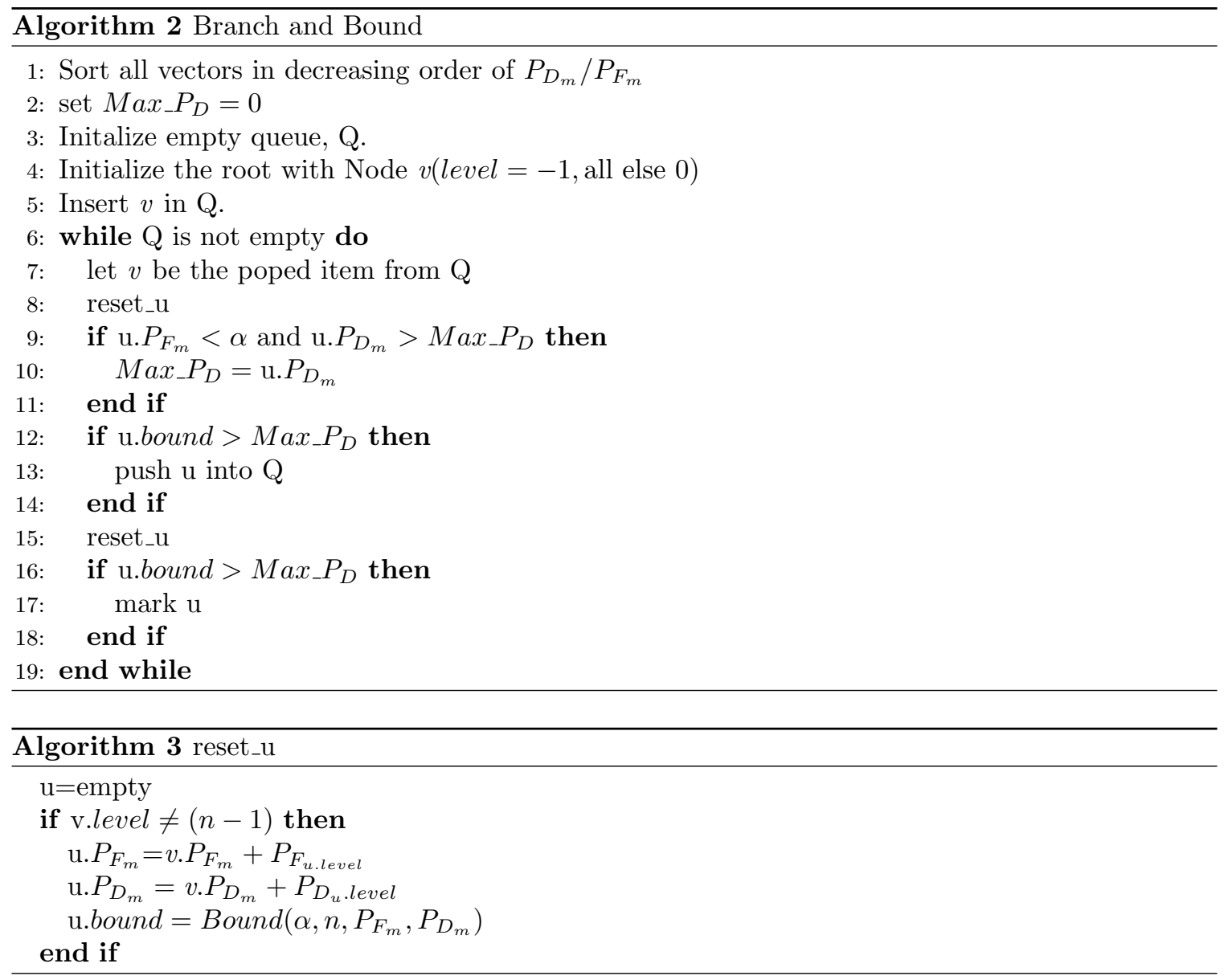

Following the step by step procedure mentioned in Algorithm 2, we can come up with the graph as shown below

In Figure 2.2, by following red coloured line we get the solution. Where $x_{i}=0$ indicate $i^{\text {th }}$ item not present in the knapsack, $x_{i}=1$ indicate $i^{\text {th }}$ item present in the knapsack. And p,w denotes profit and weight at that particular node respectively and ub denotes the upper bound estimated at that particular node.

\subsubsection{Likelihood ratio test}

For GDFP we define likelihood ratio $\mathcal{L}\left(\mathbf{u}_{m}\right)$ as follows

$$
\mathcal{L}\left(\mathbf{u}_{m}\right)=\frac{p\left(\mathbf{u}_{m} \mid H_{1}\right)}{p\left(\mathbf{u}_{m} \mid H_{0}\right)} \gtrless \eta,
$$

threshold $\eta$, is the value for which

$$
\sum_{\mathbf{u}_{m}: \mathcal{L}\left(\mathbf{u}_{m}\right)>\eta} p\left(\mathbf{u}_{m} \mid H_{0}\right)=P_{F} .
$$

To implement LRT we first calculate $\mathcal{L}\left(\mathbf{u}_{m}\right), m \in\{1, \ldots M\}$ and sort them in the accending order. When the likelihood ration $\mathcal{L}$ is big, we should accept in $H_{1}$ region. Let $[\eta, \infty)$ be the accepted 
Figure 2.2: Graph for Branch and Bound Example

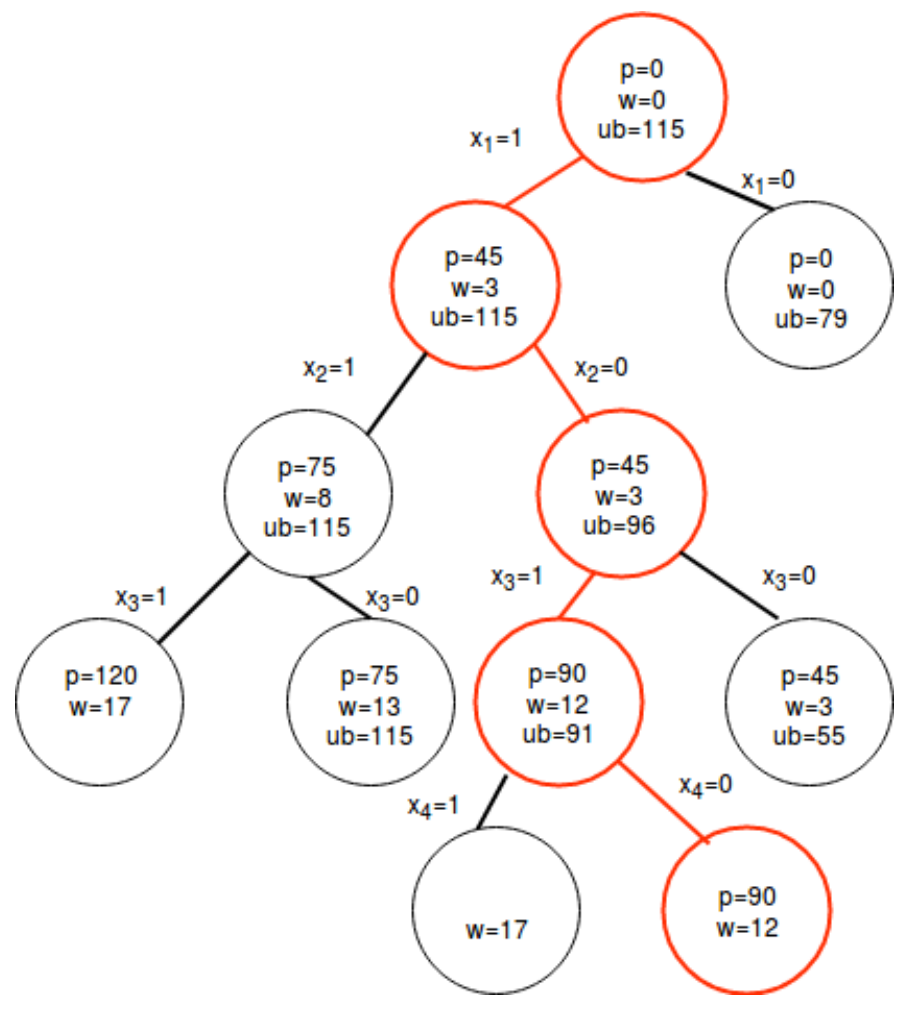

region, and $\eta$ can be obtained by $(2.10)$.

We now focus on further reducing this complexity by showing that the optimum solution $x$ is confined to a smaller dimensional observation space $\mathcal{U}^{\prime}$ in some cases, where $\left|\mathcal{U}^{\prime}\right|=2^{M^{\prime}}$ and where $M^{\prime}<M$. To facilitate this we define a desirable property namely semi-monotonic

\subsection{Semi -Monotonic Property}

Define a SU-index set (comprising of indices of the SUs) corresponding to an observation vector $\mathbf{u}_{m}$ as

$$
\mathcal{S}\left(\mathbf{u}_{m}\right)=\left\{i: u_{i, m}=1, \forall i\right\}
$$

Further define another set OV-index set (comprising of indices of the observation vectors) corresponding to an observation vector $\mathbf{u}_{m}$ as

$$
\mathbb{S}\left(\mathbf{u}_{m}\right)=\left\{m^{\prime}: \mathcal{S}\left(\mathbf{u}_{m}\right) \subsetneq \mathcal{S}\left(\mathbf{u}_{m}^{\prime}\right), \forall m^{\prime}\right\}
$$

Definition 2 :(semi-monotonic) If there exist a subset of the observation vectors on which LR is monotonic then we call such decision fusion problem as monotonic.

Under a resonable assumption $p_{f_{i}}<0.5<p_{d_{i}} \forall i$ is semi monotonic. Let us see how

Proof: The simplified form of the LRT of (2.9) is given by [16]

$$
\mathcal{L}\left(\mathbf{u}_{m}\right) \underset{x_{m}=0}{\stackrel{x_{m}=1}{\gtrless}} \eta
$$


Here in this case $\frac{p_{d_{i}}}{1-p_{d_{i}}}>1, \frac{1-p_{f_{i}}}{p_{f_{i}}}>1, \mathcal{L}()$ therfore always positive $\forall i$.

As a result, using (2.12) we get

$$
\mathcal{L}\left(\mathbf{u}_{m}\right)<\mathcal{L}\left(\mathbf{u}_{m}^{\prime}\right) \forall m^{\prime} \mathbb{S}\left(\mathbf{u}_{m}\right)
$$

And also

$$
\begin{aligned}
& p\left(\mathbf{u}_{m} \mid H_{1}\right)<p\left(\mathbf{u}_{m} \mid H_{1}\right), \forall m^{\prime} \in \mathbb{S}\left(\mathbf{u}_{m}\right), \\
& p\left(\mathbf{u}_{m} \mid H_{0}\right)>p\left(\mathbf{u}_{m} \mid H_{0}\right), \forall m^{\prime} \in \mathbb{S}\left(\mathbf{u}_{m}\right) .
\end{aligned}
$$

Figure 2.3 illustrates the semi-monotonic property exhibited by observation vectors for $\mathrm{N}=4$. The SU-index set $\mathcal{S}\left(\mathbf{u}_{t}\right)$ of the observation vector at the tail of an arbitrary arrow is the subset of the corresponding SU-index set $\mathcal{S}\left(\mathbf{u}_{h}\right)$ of the vector at the head of that arrow, i.e., $\mathcal{S}\left(\mathbf{u}_{t}\right) \subsetneq \mathcal{S}\left(\mathbf{u}_{h}\right)$, where $\mathbf{u}_{t}, \mathbf{u}_{h}$ denote the observation vectors at the tail and head of any arbitrary arrow. As an example, $\mathcal{S}([0000])=\{\}, \mathcal{S}([0001])=\{0\}^{2}, \mathcal{S}([0010])=\{1\}$ etc., and $\mathbb{S}([0001])=\{3,5,7,9,11,13,15\}$. $\mathbb{S}([1010])=\{10,13,14,15\}$ is illustrated in the figure 2.4 in red coloured boxes.

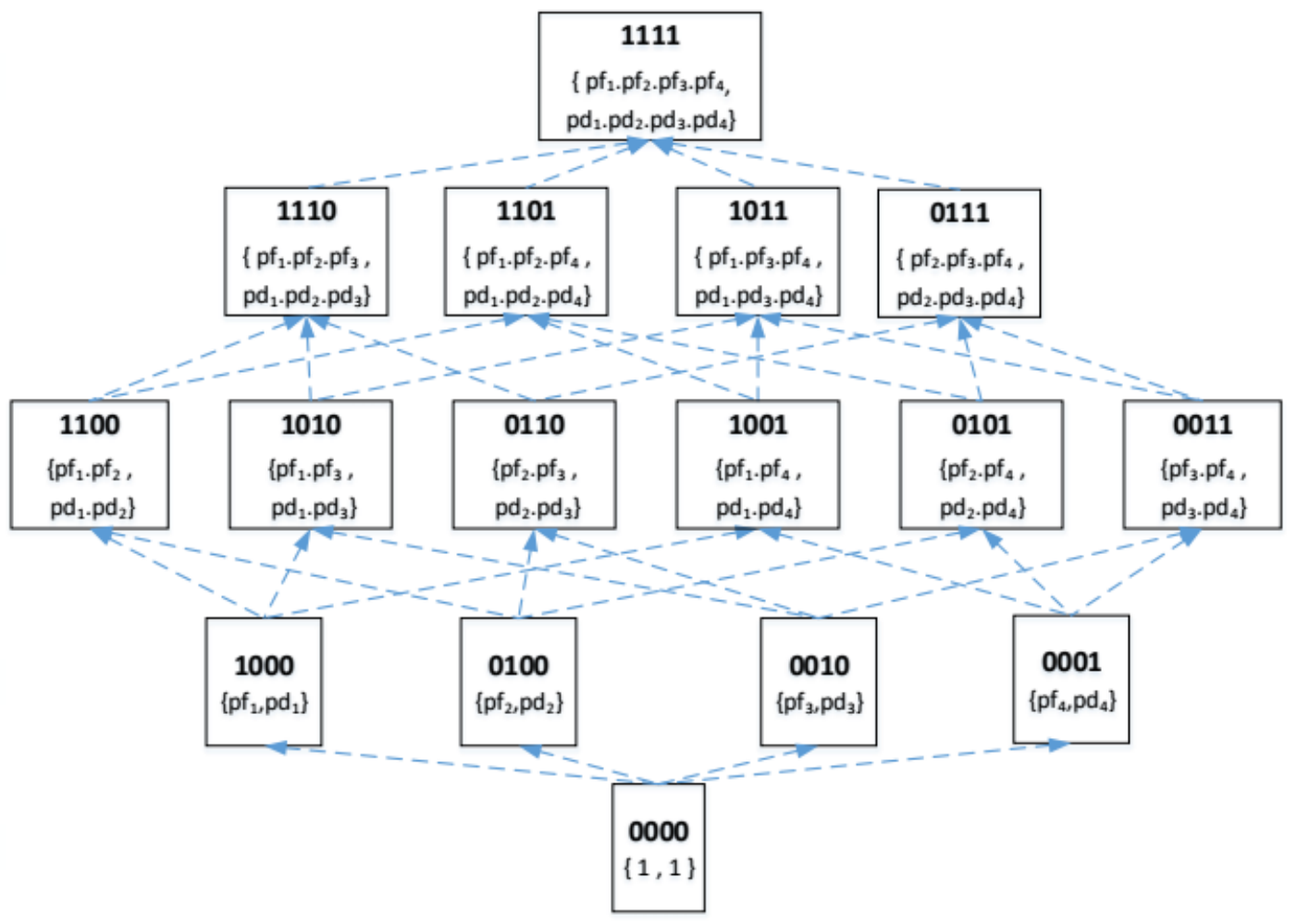

Figure 2.3: Semi-monotonic property by observation vectors for $\mathrm{N}=4$

From (2.14) and (2.15) it can be noted that, if $x_{m}=1$ in an optimal fusion rule $\mathrm{x}$, then

$$
x_{m}^{\prime}=1, \forall m^{\prime} \in \mathbb{S}\left(\mathbf{u}_{m}\right),
$$

Lemma: If $x_{m}=1$ in an optimal fusion rule, then the corresponding system probability of false 


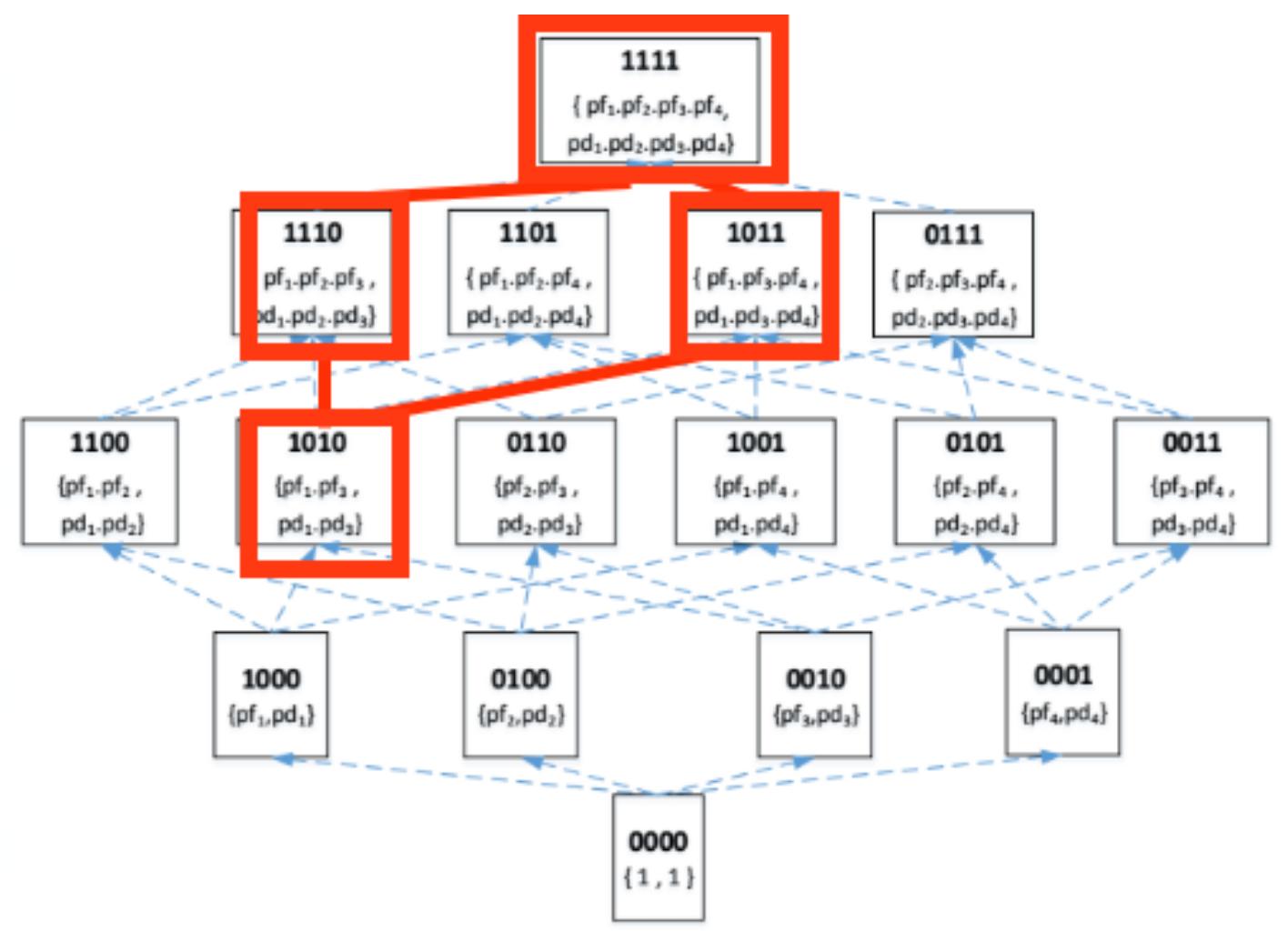

Figure 2.4: $\mathbb{S}([1010])=\{10,13,14,15\}$

alarm denoted by $P_{F}\left(x_{m}=1\right)$ is

$$
P_{F}\left(x_{m}=1\right) \geq \prod_{i \in \mathcal{S}\left(\mathbf{u}_{m}\right)} p_{f_{i}},
$$

Proof: Using (2.16), we have

$$
P_{F}\left(x_{m}=1\right) \geq p\left(\mathbf{u}_{m} \mid H_{0}\right)+p\left(\mathbf{u}_{m}^{\prime} \mid H_{0}\right), \forall m^{\prime} \in \mathbb{S}\left(u_{m}\right),
$$

Expanding and simplifying the LHS of (2.18) using (2.4), we get

$$
P_{F}\left(x_{m}=1\right) \geq \prod_{i \in \mathcal{S}\left(\mathbf{u}_{m}\right)} p_{f_{i}} .
$$

\subsection{Variable Reduction in GDFP}

we now define a reduced set of observation vector space $\mathcal{U}^{\prime}$ as

$$
\mathcal{U}^{\prime}=\left\{u_{m}: \prod_{i \in \mathcal{S}\left(\mathbf{u}_{m}\right)} p_{f_{i}} \leq \alpha, \forall m\right\},
$$

and reduced dimension $M^{\prime}=\left|\mathcal{U}^{\prime}\right|$. Note that,

- those observation vectors um that result in the system false alarm $P_{F}\left(x_{m}=1\right)$ to exceed the 
specified constraint value $\alpha$, are not included in the reduced observation space $\mathcal{U}^{\prime}$.

- those observation vectors um that result in the system false alarm $P_{F}\left(x_{m}=1\right)$ to exceed the specified con- straint value $\alpha$, are not included in the reduced observa- tion space $\mathcal{U}^{\prime}$.

- the feasible fusion solutions are now confined to the space $\mathcal{U}^{\prime}$.

- the feasible fusion solutions are now confined to the space $\mathcal{U}^{\prime}$.

- the boolean variables $x_{m}$ corresponding to the um not in the space $\mathcal{U}^{\prime}$, can now be fixed to $x_{m}=0$ (namely fixed-variable).

- to obtain the optimal $\mathrm{x}^{*}$, we now need to search the optimum value of only the remaining free-variables.

Using (2.7) the reduced variable GDFP is now defined as

$$
\operatorname{Max} \sum_{\mathbf{u}_{m} \in \mathcal{U}^{\prime}} s_{m} P_{D_{m}}, \text { sub to } \sum_{\mathbf{u}_{m} \in \mathcal{U}^{\prime}} s_{m} P_{F_{m}} \leq \alpha, s_{m} \in\{0,1\}
$$

The proposed DP-based solution can now be applied to (2.20) to obtain the optimal value of the freevariables in $\mathrm{x}$. In the following section we present the numerical results that confirm the correctness of the proposed solution and the reduced dimension $M^{\prime}$ obtained for different $N$ and $\alpha$. 


\section{Chapter 3}

\section{Numerical Results}

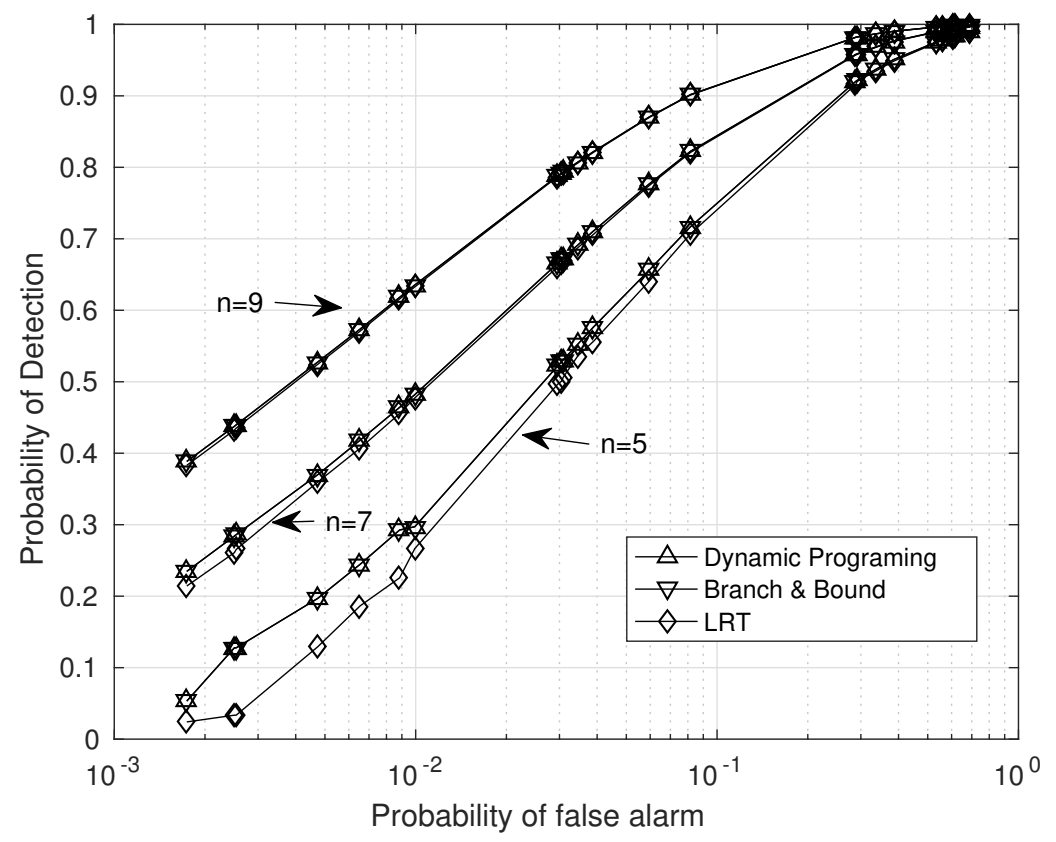

Figure 3.1: Receiver operating characteristic

To show the effectiveness of the proposed algorithm, as an example we have considered individual SUs following probability of false alarm $p_{f_{i}} \in U[0.2,0.4]$ and probability of detection $p_{d_{i}} \in U[0.6,0.8]$ where $U$ is uniform distribution. Figure 3.1 shows that the DP and BB algorithms display the same solution but LRT shows a sub-optimal solution when compaired with DP and BB. This graph was plotted by considering $0.001<\alpha<1$ and averaged the curves with 25 iteration. Figure 3.2 shows time complexity of the algorithms with $\alpha=0.05$ and averaged the curves over 1000 iteration. In the implementaion of DP we have used scaling factor $r=10^{6}$. In DP (2.7) requires $M * I_{\alpha}$ mathematical operation and to know the vector included in the solution requires $M$ operations. Hence order of complexity for DP is $\mathcal{O}\left(M * I_{\alpha}\right)$. In BB Greedy approach helps us to check particular node can give us a better solution or not, this minimizes the number of nodes that we need to travel. But in the worst case scenario, we have to travel all the nodes which makes order of complexity for BB 


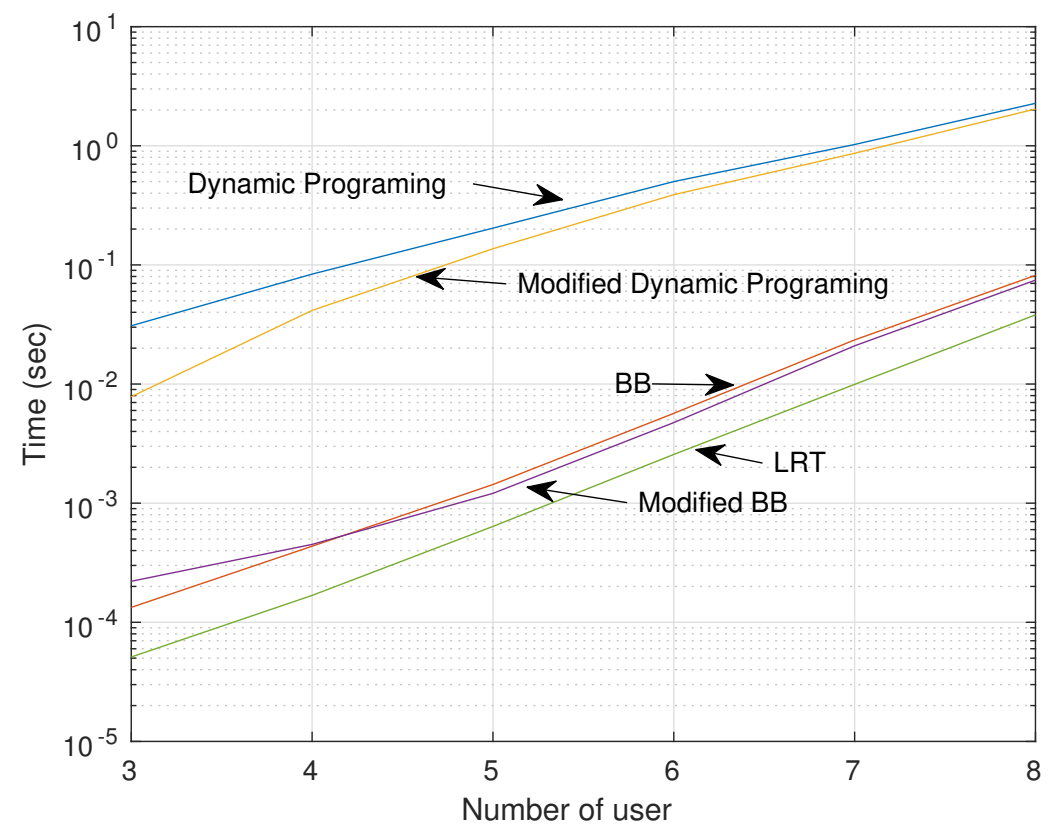

Figure 3.2: Time Complexity

$\mathcal{O}\left(2^{M}\right)$. In LRT, Sorting likelihood by Merge sort algorithm requires $\mathcal{O}(M * \log (M))$ operations and (2.10) in worst condition requires $M$ operation. Therefore, the overall complexity for LRT will be $\mathcal{O}(M * \log (M))$.In reduced variable approach, we try to decrease the number of vectors that given to $\mathrm{DP}$ or $\mathrm{BB}$ to solve GDFP problem which in return decrease the time taken to solve the problem as compared to when $\mathrm{DP}$ or $\mathrm{BB}$ are given with all the vectors. 


\section{Chapter 4}

\section{Performance improvement with Reduced Complexity Optimal Hard Decision Fusion under NP Criterion}

Distributed detection in Cognitive radio network is used to detect the presence of the primary user with the help of geographically diversly spread Secondary users (SUs). This SUs collect the necessary information and send it to Fusion centre where the further processing takes place and a better decision is made about the presence of the Primary user [20],[21],[22]. In general, the channel between SUs and FC is assumed to be noise free and fading free but in real life, it is not the same. When channel between SUs and FC is considered to be a wireless channel then the channel will definitely undergo fading and the system model would be as shown in Figure 4.1.

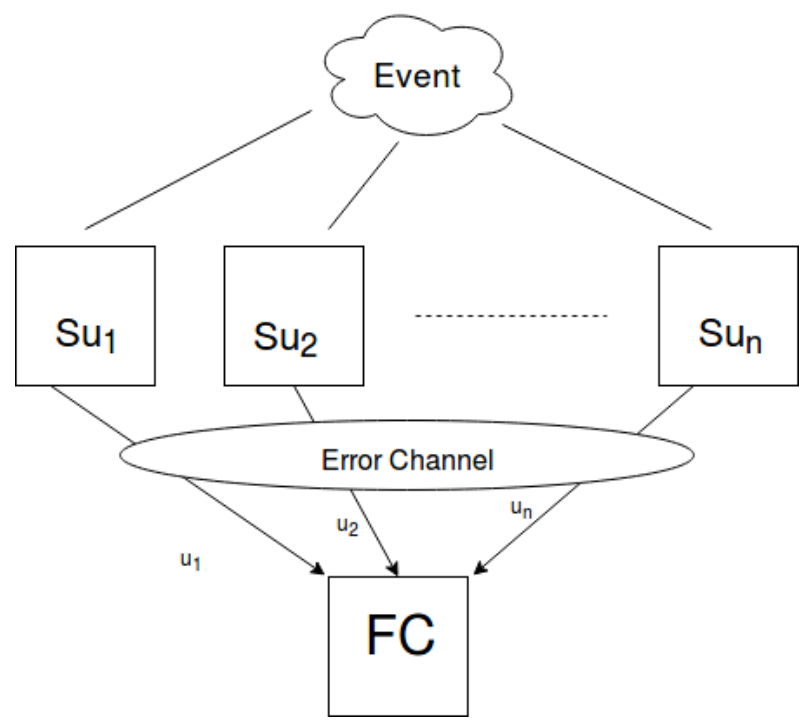

Figure 4.1: Realistic model of the system 
Each SUs send a local decision $u_{i} \in\{0,1\}$ over an erroneous channel to FC with bit probability error $p_{e_{i}} \forall i$. The channel between SUs and FC can be modelled as a binary symmetric channel[18]. Considering SUs decisions to be conditionally independent then we have

$$
\begin{aligned}
& p\left(\mathbf{u} \mid H_{1}\right)=\prod_{i=0}^{N-1}\left(p_{d_{i}}^{e}\right)^{u_{i}}\left(1-p_{d_{i}}^{e}\right)^{\left(1-u_{i}\right)}, \\
& p\left(\mathbf{u} \mid H_{0}\right)=\prod_{i=0}^{N-1}\left(p_{f_{i}}^{e}\right)^{u_{i}}\left(1-p_{f_{i}}^{e}\right)^{\left(1-u_{i}\right)},
\end{aligned}
$$

where $p_{d_{i}}^{e}=\left(1-p_{e_{i}}\right)\left(p_{d_{i}}\right)+\left(p_{e_{i}}\right)\left(1-p_{d_{i}}\right)$ and $p_{f_{i}}^{e}=\left(1-p_{e_{i}}\right)\left(p_{f_{i}}\right)+\left(p_{e_{i}}\right)\left(1-p_{f_{i}}\right)$.

\subsection{Maximal Ratio Combing}

The effect of fading over channel can be overcome using spatial diversity. where we use multiple antennas at transmitter or receiver or even both. Here we use multiple antennas at FC and single antenna at individual SUs as shown in Figure 4.2. A common architecture followed in a distributed

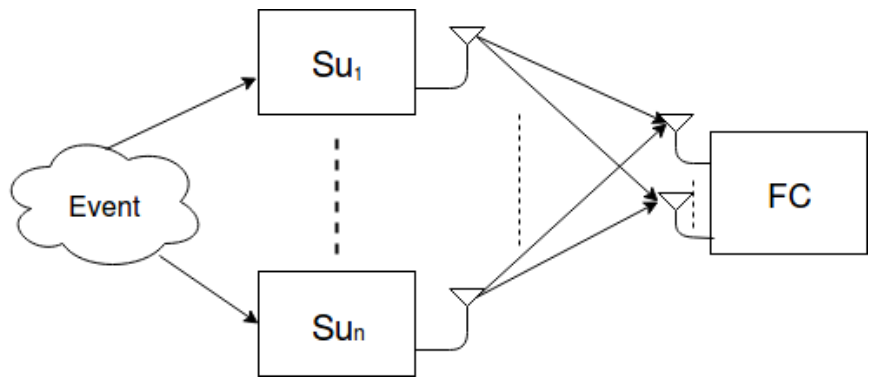

Figure 4.2: Multiple antennas at FC

detection system is parallel access channel(PAC) i.e the SUs are assigned an orthogonal channel for transmission. Here we are using the same technique to get information from the individual SUs. Since single SU is transmitting at a time we can consider single antenna transmitter and multiple antenna receiver system. For this kind of system Maximal ratio combining (MRC) can be used as a receiver.

Let us see now how MRC works. signal received from $\mathrm{SU}$ at FC will be of the $\bar{y}=\bar{h} x+\bar{n}$ where

$$
\bar{y}=\left[\begin{array}{l}
y_{1} \\
y_{2}
\end{array}\right], \bar{h}=\left[\begin{array}{l}
h_{1} \\
h_{2}
\end{array}\right], \bar{n}=\left[\begin{array}{l}
n_{1} \\
n_{2}
\end{array}\right] .
$$

Instantaneous bit-energy to noise ratio at $i_{t h}$ receiver with $h_{i}$ channel is given by

$$
\gamma=\frac{\left\|\bar{h}^{2}\right\| * E_{b}}{N_{0}},
$$

considering $h_{i} \forall i$ to be independent identically Rayleigh distributed random variable then the term $\left\|\bar{h}^{2}\right\|$ become chi-squared random variable with two degrees of freedom. Therefore the pdf of effective 
bit energy-to-noise ratio $\gamma$ can be written as

$$
p_{d f}(\gamma)=\frac{1}{(N-1) !\left(E_{b} / N_{0}\right)^{N}} \gamma^{N-1} e^{\frac{-\gamma}{\left(E_{b} / N_{0}\right)}},
$$

probability of error or BER for maximal ratio combining can be expressed as[23]

$$
\begin{gathered}
p_{e_{M R C}}=\int_{0}^{\infty} \frac{1}{2} \operatorname{erfc}(\sqrt{\gamma}) p_{d f}(\gamma) \\
p_{e_{M R C}}=\int_{0}^{\infty} \frac{1}{2} \operatorname{erfc}(\sqrt{\gamma}) \frac{1}{(N-1) !\left(E_{b} / N_{0}\right)^{N}} \gamma^{N-1} e^{\frac{-\gamma}{\left(E_{b} / N_{0}\right)}}
\end{gathered}
$$

which can be rewritten as

$$
p_{e_{M R C}}=P^{N} * \sum_{k=0}^{N-1}\left(\begin{array}{c}
N-1+k \\
k
\end{array}\right)(1-P)^{k},
$$

where

$$
P=\frac{1}{2}-\frac{1}{2}\left(1+\frac{1}{E_{b} / N_{0}}\right)^{-1 / 2}
$$

\subsection{Alamouti code}

In the literature most of the times there has been a study of using the spatial diversity between SU and FC. Now we would like to explore the space and time diversity between the SUs and FC. This can be done with the help of space-time block codes. Space-time block codes used in MIMO system to transmit the multiple copies of the same data over the erroneous channel. These codes combine all the received data in an optimal manner to extract the better information when compared to what could have been obtained by receiving only one data observation.

Space-time block codes use both space and time diversity which enables them to achieve significant gain. In Space-time block codes the data is encoded before transmission and sent on multiple antennas and also spread over time. One of the elegant methods to implement space-time block codes is MIMO Alamouti code or simply called as Alamouti code[19].

Alamouti scheme is differential space-time block code which means receiver does not need to know the channel state information to decode the data.

\subsection{2x1 Alamouti Code}

Alamouti code with two transmitters and one receive antenna is shown in figure 4.3. The encoded symbols transmitted over two antennas at first and second time slots is as follows

\begin{tabular}{|c|c|c|}
\hline & Tx1 & Tx2 \\
\hline Time T & $x_{1}$ & $x_{2}$ \\
Time T+t & $-x_{2}^{*}$ & $x_{1}^{*}$ \\
\hline
\end{tabular}


where $x_{1}$ and $x_{2}$ are modulated symbols. Let $y_{1}$ and $y_{2}$ be two received symbols at first and second time slot then we have

$$
\left[\begin{array}{l}
y_{1} \\
y_{2}^{*}
\end{array}\right]=\left[\begin{array}{cc}
h_{1} & h_{2} \\
h_{2}^{*} & -h_{1}^{*}
\end{array}\right]\left[\begin{array}{l}
x_{1} \\
x_{2}
\end{array}\right]+\left[\begin{array}{l}
n_{1} \\
n_{2}
\end{array}\right]
$$

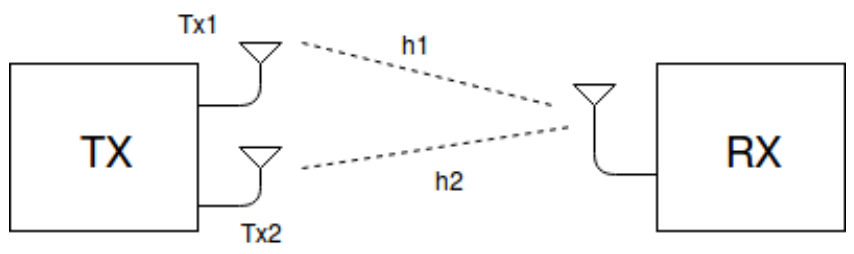

Figure 4.3: 2x1 Alamouti scheme

and $h_{1}$ is the channel from first transmit antenna to receive antenna, $h_{2}$ is the channel from second transmit antenna to receive antenna, and $n_{1}, n_{2}$ are the noise at time slot 1 and time slot 2. Further,

$$
\bar{y}=\overline{c_{1}} x_{1}+\overline{c_{2}} x_{2}+\bar{n}
$$

where

$$
\overline{c_{1}}=\left[\begin{array}{l}
h_{1} \\
h_{2}^{*}
\end{array}\right], \overline{c_{2}}=\left[\begin{array}{c}
h_{2} \\
-h_{1}^{*}
\end{array}\right]
$$

since $\overline{c_{1}}, \overline{c_{2}}$ are orthogonal,alamouti code is also known as orthogonal space time code. After the simplification of (4.5) we get

$$
\frac{\overline{c_{1}} H}{\left\|\overline{c_{1}}\right\|} y=\left\|\overline{c_{1}}\right\| x+\tilde{n}
$$

Therefore the SNR of $2 \times 1$ alamouti can be written as

$$
S N R=\frac{\left\|\bar{h}^{2}\right\| * E_{b}}{2 * N_{0}}
$$

we know $h_{i} \forall i$ to be independent identically Rayleigh distributed random variable then the term $\left\|\bar{h}^{2}\right\|$ becomes chi-squared random variable with two degrees of freedom. similar to that of MRC probability of error or bit-error of 2x1 Alamouti scheme can be derived as

$$
p_{e_{\text {Alamout } 2 x 1}}=P_{\text {Alamout }}^{2}\left[1+2\left(1-P_{\text {Alamout }}\right)\right]
$$

where

$$
P_{\text {Alamouti }}=\frac{1}{2}-\frac{1}{2}\left(1+\frac{2}{E_{b} / N_{0}}\right)^{-1 / 2}
$$

\section{$4.42 \times 2$ Alamouti Code}

In this scheme we use two transmit and two receive antenna as shown in Figure 4.4.

The encoded symbols transmitted over two antennas at first and second time slot is as follows

\begin{tabular}{|c|c|c|}
\hline & Tx1 & Tx2 \\
\hline Time T & $x_{1}$ & $x_{2}$ \\
Time $\mathrm{T}+\mathrm{t}$ & $-x_{2}^{*}$ & $x_{1}^{*}$ \\
\hline
\end{tabular}




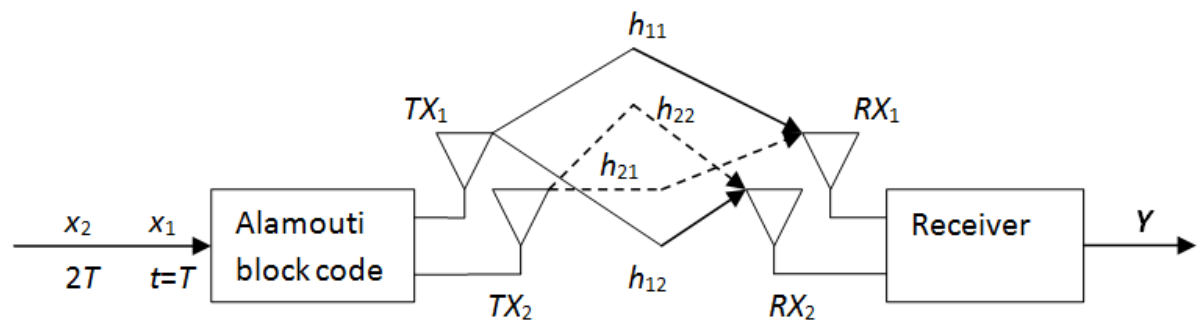

Figure 4.4: 2x2 Alamouti scheme

where $x_{1}$ and $x_{2}$ are modulated symbols.

At the receiver during first time slot and second time we receive the symbols as follows

$$
\begin{aligned}
& {\left[\begin{array}{l}
y_{11} \\
y_{12}
\end{array}\right]=\left[\begin{array}{ll}
h_{11} & h_{12} \\
h_{21} & h_{22}
\end{array}\right]\left[\begin{array}{l}
x_{1} \\
x_{2}
\end{array}\right]+\left[\begin{array}{l}
n_{11} \\
n_{12}
\end{array}\right],} \\
& {\left[\begin{array}{l}
y_{21} \\
y_{22}
\end{array}\right]=\left[\begin{array}{ll}
h_{11} & h_{12} \\
h_{21} & h_{22}
\end{array}\right]\left[\begin{array}{c}
-x_{2}^{*} \\
x_{1}^{*}
\end{array}\right]+\left[\begin{array}{l}
n_{21} \\
n_{22}
\end{array}\right],}
\end{aligned}
$$

where $y_{11}, y_{12}, y_{21}$ and $y_{22}$ represent antenna 1 at first time slot, by antenna 1 at second time slot, by antenna 2 at first time slot, and by antenna 2 at second time slot. $h_{i j}$ denotes the channel coefficient of $i^{\text {th }}$ receiver and $j^{\text {th }}$ transmitter. And $\left[\begin{array}{l}n_{11} \\ n_{12}\end{array}\right]$ recevier noise at antenna 1 and antenna 2 during time slot 1 and $\left[\begin{array}{l}n_{21} \\ n_{22}\end{array}\right]$ recevier noise at antenna 1 and antenna 2 during time slot 2. combing both time slots we get

$$
\left[\begin{array}{l}
y_{11} \\
y_{12} \\
y_{21}^{*} \\
y_{22}^{*}
\end{array}\right]=\left[\begin{array}{cc}
h_{11} & h_{12} \\
h_{21} & h_{22} \\
h_{12}^{*} & -h_{11}^{*} \\
h_{22}^{*} & -h_{21}^{*}
\end{array}\right]\left[\begin{array}{l}
x_{1} \\
x_{2}
\end{array}\right]+\left[\begin{array}{l}
n_{11} \\
n_{12} \\
n_{21} \\
n_{22}
\end{array}\right]
$$

from equation(4.11)we define $H$ matrix as

$$
H=\left[\begin{array}{cc}
h_{11} & h_{12} \\
h_{21} & h_{22} \\
h_{12}^{*} & -h_{11}^{*} \\
h_{22}^{*} & -h_{21}^{*}
\end{array}\right]
$$

using pseudo inverse of a matrix $H$ we can estimate the transmitted symbol as

$$
\left[\begin{array}{l}
\hat{x_{1}} \\
\hat{x_{2}}
\end{array}\right]=\left(H^{H} H\right)^{-1} H^{H}\left[\begin{array}{l}
y_{11} \\
y_{12} \\
y_{21}^{*} \\
y_{22}^{*}
\end{array}\right]
$$

To calculate BER or Probability of error from equation (4.12) which will be similar to calculation of 
probability of error for MRC with four receiver with slight modification[23]. Finally the probability of error for $2 \times 2$ alamouti code given as follows

$$
p_{e_{\text {Alamout } 2 \times 1} 1}=P_{\text {Alamouti }}^{4}\left[1+4\left(1-P_{\text {Alamout } i}\right)+10\left(1-P_{\text {Alamout } i}\right)^{2}+20\left(1-P_{\text {Alamout } i}\right)^{3}\right]
$$

where

$$
P_{\text {Alamouti }}=\frac{1}{2}-\frac{1}{2}\left(1+\frac{2}{E_{b} / N_{0}}\right)^{-1 / 2}
$$




\section{Chapter 5}

\section{Numerical Results}

The Figure 5.1 shows how Probability of detection $(\mathrm{Pd})$ varies with respect to Signal to Noise Ratio(SNR) by using the SNR enhancement methods that have been discussed in the previous chapter at FC to improve the reception quality of data that is sent by SUs over the erroneous channel. To plot this graph we have considered individual SUs with following probability of false alarm $p_{f_{i}} \in U[0.2,0.4]$ and probability of detection $p_{d_{i}} \in U[0.6,0.8]$ where $U$ is uniform distribution.With allowed total Probability of false alarm $\alpha=0.1$. For number of SUs $n=5,7$ and 11 .

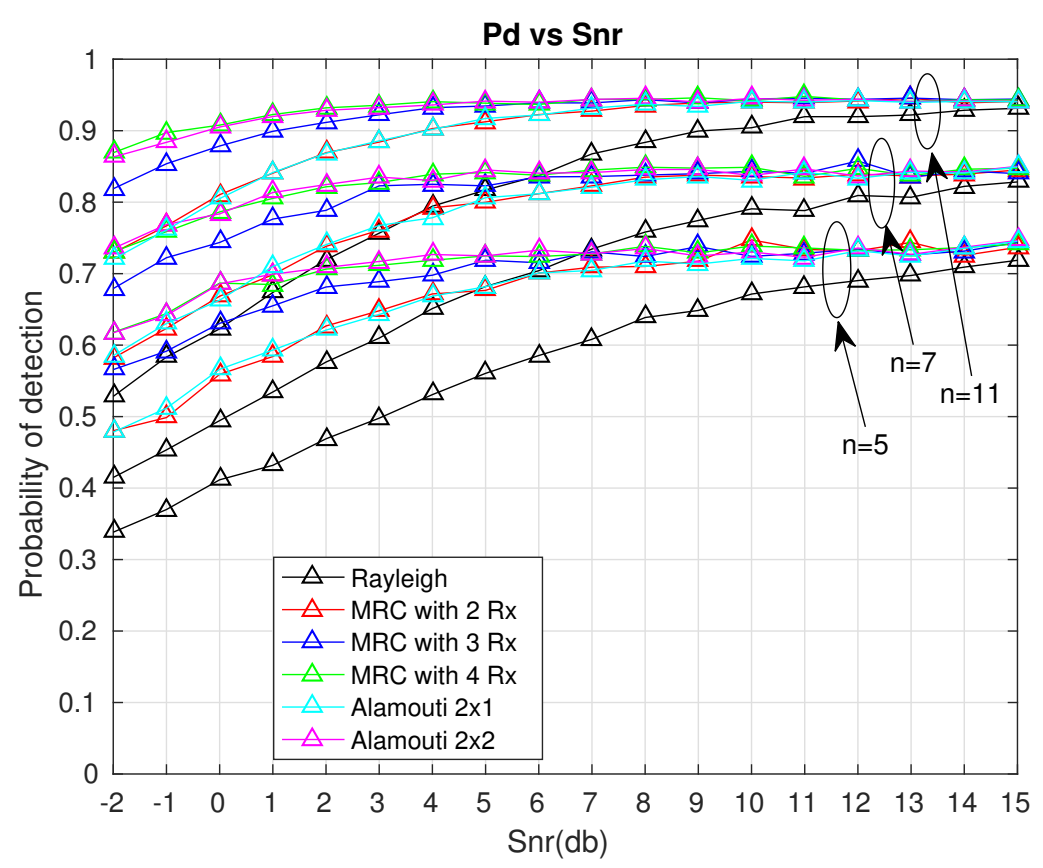

Figure 5.1: Pd vs SNR

For Rayleigh fading channel without using any performance enhancement methods we have considered probability of error $p_{e}=\frac{1}{2}\left(1-\sqrt{\frac{S N R}{2+S N R}}\right)$.We have used MRC at FC with 2,3 and 4 receive antennas and single transmit antenna at SU and using() for probability of error. Whereas for alamouti scheme of performance enhancement we have considered two cases. In the first case with 
2 transmit antenna and 1 receive antenna and second case uses 2 transmit and 2 receive antenna. And use (4.8) and (4.14) as probability of error equations.

The above mentioned methods are helpful in improving the data vectors received at FC and the Global decision making is done by variable reduction with dynamic programming to solve GDFP problem. 


\section{Chapter 6}

\section{Conclusion}

We have utilized the semi-monotonic property exhibited in a special case of decision fusion problem to decrease the dimensions in the feasible solution space. Eventually, we apply dynamic programming to solve the problem with further reduced complexity. Further, we have seen the effect of using multiple antennas schemes such as MRC and Alamouti code at FC with reduced complexity rule. 


\section{References}

[1] P. K. Varshney, Distributed Detection and Data Fusion. Springer Science \& Business Media, 1997.

[2] R. Viswanathan and P. K. Varshney, Distributed Detection with Multiple Sensors: Part IFundamentals, Proc. IEEE, vol. 85, no. 1, pp. 54-63, Jan. 1997

[3] J. Mitola and G. Q. Maguire, Cognitive Radio: Making Software Radios More Personal, IEEE Personal Commun. Mag., vol. 6, no. 4, pp. 13-18, Aug. 1999.

[4] D. Nikhil, M. F. Rahaman and M. Z. A. Khan, Reduced Complexity Optimal Hard Decision Fusion under Neyman-Pearson Criterion, 26th IEEE SIU 2018 conference, Turkey

[5] S. Haykin, Cognitive Radio: Brain-Empowered Wireless Communica- tions, IEEE Journal on Selected Areas in Communications, vol. 23, no. 2, pp. 201-220, Feb. 2005.

[6] J. Lunden, V. Koivunen, and H. V. Poor, Spectrum Exploration and Exploitation for Cognitive Radio: Recent Advances, IEEE Signal Processing Magazine, vol. 32, no. 3, pp. 123-140, May 2015.

[7] E. Axell, G. Leus, E. G. Larsson, and H. V. Poor, Spectrum Sensing for Cognitive Radio : Stateof-the-Art and Recent Advances, IEEE Signal Processing Magazine, vol. 29, no. 3, pp. 101-116, May 2012.

[8] K. B. Letaief and W. Zhang, Cooperative Communications for Cog- nitive Radio Networks, Proceedings of the IEEE, vol. 97, no. 5, pp. 878-893, May 2009.

[9] Z. Quan, S. Cui, H. V. Poor, and A. H. Sayed, Collaborative Wideband Sensing for Cognitive Radios, IEEE Signal Processing Magazine, vol. 25, no. 6, pp. 60-73, Nov. 2008.

[10] S. M. Mishra, A. Sahai, and R. W. Brodersen, Cooperative Sensing among Cognitive Radios, in 2006 IEEE International Conference on Communications, vol. 4, pp. 1658-1663, Jun. 2006.

[11] Poor, H. Vincent. An introduction to signal detection and estimation. Springer Science \& Business Media, 2013.

[12] Lehmann, Erich Leo. "Testing Statistical Hypotheses (Springer Texts in Statistics)." (1997).

[13] M. F. Rahaman and M. Z. A. Khan, Low-Complexity Optimal Hard Decision Fusion Under the Neyman-Pearson Criterion, IEEE Signal Process. Lett., vol. 25, no. 3, pp. 353-357, Mar. 2018. 
[14] E. Demaine and S. Devadas. (2011, Fall) 6.006 Introduction to Algorithms. Video Lectures, MIT OpenCourseWare, License: Creative Commons BY-NC-SA. Massachusetts Institute of Technology. [Online]. Available: https://ocw.mit.edu

[15] Rust, John. "Numerical dynamic programming in economics." Handbook of computational economics 1 (1996): 619-729.

[16] Z. Chair and P. K. Varshney, Optimal Data Fusion in Multiple Sensor Detection Systems, IEEE Trans. Aerosp. Electron. Syst., vol. AES-22, no. 1, pp. 98-101, Jan. 1986.

[17] Chen, B., Tong, L. and Varshney, P.K., 2006. Channel-aware distributed detection in wireless sensor networks. IEEE Signal Processing Magazine, vol.23, no.4, pp.16-26, 2006.

[18] Ciuonzo, D., Romano, G. and Rossi, P.S., Channel-aware decision fusion in distributed MIMO wireless sensor networks: Decode-and-fuse vs. decode-then-fuse. IEEE transactions on wireless communications, vol. 11, no. 8, pp.2976-2985, 2012.

[19] Alamouti, S.M., A simple transmit diversity technique for wireless communications. IEEE Journal on selected areas in communications, vol. 16,no. 8, pp.1451-1458, 2012.

[20] Berger, Christian R., Marco Guerriero, Shengli Zhou, and Peter Willett. "PAC vs. MAC for decentralized detection using noncoherent modulation." IEEE Transactions on Signal Processing, vol. 57, no. 9, pp. 3562-3575, 2009.

[21] Banavar, Mahesh K., Anthony D. Smith, and Andreas Spanias. "Distributed detection over fading MACs with multiple antennas at the fusion center." In Acoustics Speech and Signal Processing (ICASSP), IEEE International Conference on, pp. 2894-2897. IEEE, 2010.

[22] Zhang, Xin, H. Vincent Poor, and Mung Chiang. "Optimal power allocation for distributed detection over MIMO channels in wireless sensor networks." IEEE Transactions on Signal Processing,vol. 56, no. 9 , pp. 4124-4140, 2008.

[23] Rao, K. Deergha. Channel coding techniques for wireless communications. Springer, 2016. 